\title{
Social-Aware VR Configuration Recommendation via Multi-Feedback Coupled Tensor Factorization
}

\author{
Hsu-Chao Lai \\ Dept. of Computer Science, National \\ Chiao Tung Univ. \\ Inst. of Information Science, \\ Academia Sinica, Taiwan \\ hclai0806@iis.sinica.edu.tw \\ Jiun-Long Huang \\ Dept. of Computer Science, National \\ Chiao Tung Univ., Taiwan \\ jlhuang@cs.nctu.edu.tw
}

\author{
Hong-Han Shuai \\ Dept. of Electrical and Computer \\ Engineering, National Chiao Tung \\ Univ., Taiwan \\ hhshuai@nctu.edu.tw
}

\author{
Wang-Chien Lee \\ Dept. of Computer Science and \\ Engineering, The Pennsylvania State \\ Univ., USA \\ wlee@cse.psu.edu
}

\author{
De-Nian Yang \\ Inst. of Information Science \\ Research Center for Information \\ Technology Innovation \\ Academia Sinica, Taiwan \\ dnyang@iis.sinica.edu.tw \\ Philip S. Yu \\ Shanghai Inst. for Advanced \\ Communication and Data Science, \\ Shanghai Key Laboratory of Data \\ Science, Fudan Univ. Computer \\ Science Dept., Univ. of Illinois at \\ Chicago, USA \\ psyu@uic.edu
}

\begin{abstract}
Recent technological advent in virtual reality (VR) has attracted a lot of attention to the VR shopping, which thus far is designed for a single user. In this paper, we envision the scenario of $V R$ group shopping, where VR supports: 1) flexible display of items to address diverse personal preferences, and 2) convenient view switching between personal and group views to foster social interactions. We formulate the Multiview-Enabled Configuration Recommendation (MECR) problem to rank a set of displayed items for a VR shopping user. We design the Multiview-Enabled Configuration Ranking System (MEIRS) that first extracts discriminative features based on Marketing theories and then introduces a new coupled tensor factorization model to learn the representation of users, MultiView Display (MVD) configurations, and multiple feedback with content features. Experimental results manifest that the proposed approach outperforms personalized recommendations and group recommendations by at least $30.8 \%$ in large-scale datasets and $63.3 \%$ in the user study in terms of hit ratio and mean average precision.
\end{abstract}

\section{KEYWORDS}

Virtual reality group shopping; configuration recommendation; Multi-View Display; coupled tensor factorization

\section{ACM Reference Format:}

Hsu-Chao Lai, Hong-Han Shuai, De-Nian Yang, Jiun-Long Huang, WangChien Lee, and Philip S. Yu. 2019. Social-Aware VR Configuration Recommendation via Multi-Feedback Coupled Tensor Factorization. In The 28th

Permission to make digital or hard copies of all or part of this work for personal or classroom use is granted without fee provided that copies are not made or distributed for profit or commercial advantage and that copies bear this notice and the full citation on the first page. Copyrights for components of this work owned by others than ACM must be honored. Abstracting with credit is permitted. To copy otherwise, or republish to post on servers or to redistribute to lists, requires prior specific permission and/or a fee. Request permissions from permissions@acm.org.

CIKM '19, November 3-7, 2019, Beijing, China

(C) 2019 Association for Computing Machinery.

ACM ISBN 978-1-4503-6976-3/19/11 . \$ \$15.00

https://doi.org/10.1145/3357384.3357952

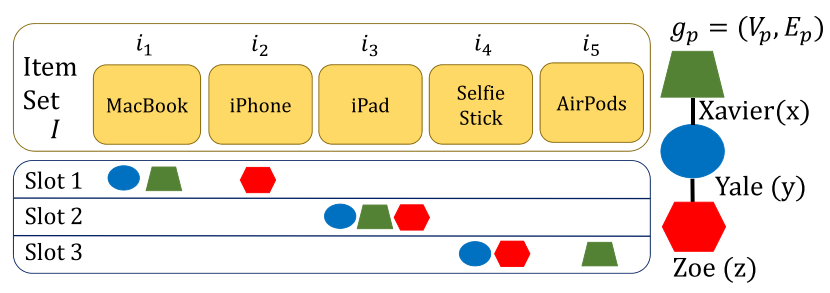

(a) Displayed items in an MVD configuration.

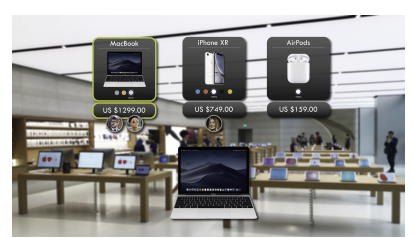

(b) Yale's primary view in slot 1 .

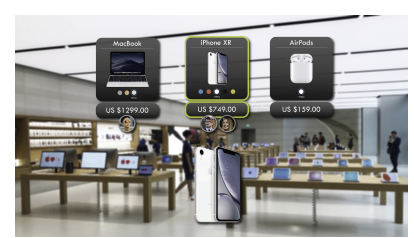

(c) Yale's group view after switching to join Zoe's view in slot 1.
Figure 1: An illustrative example of an MVD configuration.

ACM International Conference on Information and Knowledge Management (CIKM '19), November 3-7, 2019, Beijing, China. ACM, New York, NY, USA, 10 pages. https://doi.org/10.1145/3357384.3357952

\section{INTRODUCTION}

Recent technological advent in virtual reality (VR) has attracted a lot of attention to the uprising VR shopping, as demonstrated in IKEA VR Store, Alibaba Buy+, eBay and Myer's VR store, and Amazon's VR kiosks. ${ }^{1}$ Existing VR shopping solutions are designed for a single user. Without taking group shopping into consideration, the displayed items are not configured to stimulate social interactions and discussions, not to mention the potential increase in sales.

${ }^{1}$ IKEA: https://youtu.be/5_znFPj5Lis, Alibaba: https://goo.gl/cAZELG, eBay: https: //youtu.be/yAuiXhJPnr8, and Amazon: https://youtu.be/J5NviNVdOsc 
In contrast to web-based E-commerce, several VR functions can be exploited for VR group shopping. 1) Social Interaction. Users in VR can interact with each other via avatars. ${ }^{2}$ It is encouraging to support social functions in VR shopping since research demonstrates that social interactions boost sales [37]; moreover, shopping together with friends improves relationships [31]. 2) Multi-View Display (MVD). Similar to VR group traveling and gaming, the virtual environments for all VR users are not necessary to be identical in a group shopping event, since MVD supports flexible view switch between the primary view (viewing different items privately) and the group view (viewing common items with friends) during shopping $[13,16] .{ }^{3}$ Therefore, although the shopping layout and the user interface are consistent, the displayed items can be slightly tailored for different users in a group based on their diverse individual preferences.

Figure 1(a) illustrates an example of an MVD configuration. The social graph of three users, Xavier, Yale, and Zoe, is shown at the right. An item set $I$ at the top includes five products. The items displayed in three slots for each user are illustrated at the bottom. For example, at the first slot, Yale and Xavier see a MacBook, while Zoe views an iPhone in their respective primary views. Figure 1(b) shows the primary view of Yale with MacBook displayed, whereas his friends and their viewing products are listed on the right of the MacBook. If he decides to join Zoe's view on iPhone for discussion, he may press the icon of $i$ Phone to switch from his primary view to the group view as shown in Figure 1(c). With MVD, VR users are not tied to commonly displayed products, as the views can be flexibly switched to address personal preferences.

Towards the vision of VR group shopping where the personal preferences and social interactions are factored in, we formulate a new recommendation problem, namely Multiview-Enabled Configuration Recommendation (MECR), as follows. An MVD configuration is comprised of sequences of displayed items for all users, where each sequence contains $k$ ordered items. Given the feedback observed from VR shopping, e.g., view, take, discuss, and buy, the goal of MECR is to quantify the MVD personal satisfaction (considering personal interests, social interactions, and subgroup changes) of an MVD configuration and the corresponding group simultaneously.

To quantify the MVD personal satisfaction of the candidate MVD configurations and companions, who are also participating the shopping event, for VR group shopping, one possible approach is to exploit existing personalized recommendation $[10,17]$ for each individual user. However, personalized recommendation does not consider social influence during shopping. While there exists some social-aware personalized recommendation [39] inferring personal preferences with social links, it is not designed to consider the satisfaction with social interactions during shopping. On the other hand, an alternative idea is group recommendation $[2,21]$ which nevertheless presents the same configuration to all users and thus fails to address diverse individual needs. Social group query selects a group of socially tight users to foster good social atmosphere $[26,33]$ and preferred items could be recommended simultaneously [25]. However, the chosen items can only be identical for each user.

\footnotetext{
${ }^{2}$ See https://vrchat.net/ as an example.

${ }^{3}$ See IKEA in footnote 1 as an example. The displayed furniture can be switched based on user preferences (at 0:40).
}

Indeed, MECR faces new research challenges. 1) How to deal with the cold start configurations? Although heterogeneous feedback on various interactions (e.g., view, take, discuss, and buy) observed from VR shopping can be used to learn representations of users and configurations, it is challenging to derive the latent representation for new configurations due to the lack of feedback. 2) How to quantify the measure of satisfaction without ground truth? 3) Since users are partitioned into different subgroups corresponding to different slots based on their primary views, how to model the potential impact on user satisfaction due to subgroup changes?

In this paper, we propose a two-phase ranking framework Multiview-Enabled Configuration Ranking Syystem (MEIRS) to address the above challenges. Specifically, for the first challenge, a simple approach is to linearly aggregate the embeddings of users and items and then use separate neural networks to predict the user satisfaction corresponding to different feedback. The average score of these networks is returned for use. However, a more sophisticated approach is to take into account the interplay of heterogeneous feedback to generate better embeddings for satisfaction prediction. For example, "discuss" may cause "purchase", but "view" without "take" may imply dissatisfaction. Therefore, we exploit tensor factorization, as tensor is a natural mechanism to store user, configuration, and heterogeneous feedback data, to jointly learn the embeddings of users, MVD configurations, and feedback. As directly factorizing the tensor usually incurs the cold-start problem for new configurations, a naïve approach is to extract and concatenate content-based features (e.g., item correlations and 3D shape embeddings that describe item appearances) of configurations to the embeddings from tensor factorization. However, the concatenation of features may drop the correlations between two feature spaces. We further introduce a new coupled tensor factorization model, named Configuration Feature Coupled Tensor Factorization (CECON), to integrate the two latent features into a single shareable latent feature space, such that the shared latent features consist of the information from both heterogeneous feedback and content features. Moreover, the embeddings of new configurations can be derived from their content-based features.

For the second challenge, due to the lack of ground truth on user satisfaction (i.e., ratings), we introduce the notion of configuration dominance to effectively label the relative order of configuration pairs based on the discrepancy in each feedback, and employ the Bayesian Personalized Ranking (BPR) framework [22] to minimize the total pairwise ranking loss for learning user satisfaction. Furthermore, we propose foint $\underline{C} E C O N$ and Configuration Ranking (JOCOR) to jointly minimize the tensor factorization loss and the pairwise ranking loss, whereas the heterogeneous feedback, discriminative features, and the configuration dominance are carefully examined simultaneously. Moreover, inspired by self-paced learning [38], we identify different levels of dominance between paired instances to put emphasis on those paired instances with larger differences in feedback. For example, it is more critical to correctly rank a pair of configurations with a difference of 10 touches than another pair with a difference of merely 1 touch.

For the last challenge, subgroup changes between slots may interrupt the immersive experience. Moreover, according to Psychology study [4], introverts who are shy and quiet are more sensitive to 
group dynamics because they need more time to get used to new environments. For example, user A was viewing identical items with three friends in a slot and then she shares items with another three friends in the next slot. What's worse, if $\mathrm{A}$ is an introvert, she may be overwhelmed with frequent and large group dynamics in the two slots. Hence, we measure the group dynamic along the slots and extraversion for each user, where users with low extraversion implies that they are introverts, from 3D logged behavior data. We design a regularization term Group Dynamic Adoption (GDA) to penalize the satisfaction of introverts for dramatic subgroup changes. The contributions of this paper are summarized as follows.

- We identify an emerging scenario of VR group shopping, where users can enjoy social interactions together and view distinct items in their respective views by leveraging MVD. Accordingly, we formulate the MECR problem to quantify MVD personal satisfaction for each user.

- We propose a novel machine learning framework MEIRS to solve MECR. We design a new coupled tensor factorization model CECON that not only learns the embeddings of users and configurations from heterogeneous feedback but also integrates content-based features extracted from 3D logged data to alleviate the cold-start issue.

- We introduce a new notion configuration dominance to address the lack of ground truth regarding user satisfaction. We identify the importance of each instance and design JOCOR to jointly learn the embeddings from CECON and a $\mathrm{BPR}$ ranking loss weighted by the importance. A regularization term GDA is designed to penalize the satisfaction of introverts for dramatic subgroup changes.

- We conduct large-scale experiments on three real datasets for simulation of VR shopping and build VR user study systems to collect real user feedback. Experimental results manifest that MEIRS outperforms state-of-the-art personalized and group recommendations for VR group shopping by at least $30.8 \%$ in large-scale datasets and $63.3 \%$ in the user study in terms of the hit ratio and the mean average precision.

\section{RELATED WORK}

Group recommendation [2, 21] aggregates features among users in a group as group features to find group consensus. For example, Parambath et al. [21] propose to greedily select items with high consensus scores, which are derived by aggregating users' preferences in the group, and the affinity between the items. Attention networks are employed to learn a weighted sum of personal preferences to form the group embedding [2,35], such that group consensus can be retrieved. Nevertheless, group consensus fails to address diverse personal interests in MVD. An alternative approach for group recommendation is to partition users into subgroups based on their preferences [23]. However, ignoring the social relations among the group members may put close friends into different subgroups, missing an opportunity to establish the social atmosphere desirable for group shopping. Moreover, group dynamics are not optimized according to the extraversion for different users.

Personalized recommendation has been widely deployed in webbased E-commerce $[6,15]$. He et al. use category information to learn hierarchical embeddings for recommending clothing [10].
Item structure, textual content, and tag information [8] are adopted to improve recommendation. Ding et al. integrate the view and purchase logs to improve the recommendation results [6]. Conditional probabilities of feedback transformation are used to provide insight into user behaviors [29]. A graph-based embedding model is designed to recommend a social event and partners for a user jointly [34]. However, without MVD configurations, these methods focus on recommending items to individuals without encouraging social interactions. Moreover, the personal interests between users could be diverse, and these personalized recommenders fail to address the interplay of displaying identical or different items in a slot. Moreover, group dynamics are not examined.

\section{MULTI-VIEW DISPLAY CONFIGURATION RECOMMENDATION}

The goal of the proposed MEIRS is to jointly rank candidate MVD configurations and the group members for each VR group shopping user. However, it is challenging to rank the MVD configurations due to 1) cold-start issue in configurations, 2) no/few ground truth for MVD personal satisfaction, and 3) complicated subgroup changes during group shopping. To address the first challenge, the first phase of MEIRS extracts item-level and configuration-level features since the contents are available for any configuration. However, directly concatenating these content-based features with configuration embeddings derived from tensor factorization may drop the correlation between two feature spaces. Hence, the second phase of MEIRS introduces a new coupled factorization model CECON to learn the representations of users and MVD configurations simultaneously according to heterogeneous feedback coupled with various features. For the second challenge, an intuition is to exploit the number of purchase for each configuration for ranking. Nevertheless, users can enjoy shopping process without any purchase. We introduce the notion of configuration dominance for construction of proxy labels with heterogeneous feedback in place of MVD personal satisfaction, as well as identifying the importance of each training data. For the third challenge, a simple approach is to penalize the subgroup changes for all users, but users with different personalities may have different perception on these changes [4]. Therefore, a social-aware regularization term, namely, Group Dynamic Adoption (GDA), is introduced to capture the social norm of introverts and extroverts towards subgroup changes between slots according to Psychology research [4].

In the following, bold uppercase letters (e.g., $\mathrm{X}$ ) and lowercase letters (e.g., $\mathbf{x})$ denote matrices and column vectors, respectively. $\mathrm{X}(i, j)$ and $\mathrm{X}(i,:)$ denote the element in the $i$-th row and the $j$-th column and the $i$-th row vector, respectively. Non-bold letters (e.g., $x$ ) and squiggle letters (e.g., $\mathcal{X}$ ) represent scalars and tensors, respectively. Given a social network $G=(V, E)$ with $N$ users $\left\{u_{1}, u_{2}, \cdots, u_{N}\right\}$, an item set $I=\left\{i_{1}, i_{2}, \cdots, i_{l}\right\}$ with $l$ items, a shopping group set $G_{H}=\left\{g_{1}, \cdots, g_{p}, \cdots, g_{M}\right\}$ with $M$ groups, and their corresponding visited MVD configurations $C_{H}=\left\{\mathrm{C}_{1}, \cdots, \mathrm{C}_{p}, \cdots, \mathrm{C}_{M}\right\}$, where $g_{p}=\left\{V_{p}, E_{p}\right\}$ is an induced subgraph of $G$. $\mathrm{C}_{p} \in I^{\left|V_{p}\right| \times k}$ is represented as a matrix, where $\mathrm{C}_{p}(u, i)$ in $\mathrm{C}_{p}$ is the item that user $u$ views at slot $i$. In addition, heterogeneous feedback of various interactions (e.g., view, take, discuss, buy) is recorded by a tensor 
$\mathcal{T} \in \mathbb{R}^{N \times M \times D}$, where an element $\mathcal{T}(n, p, d)$ indicates the total number of interactions for feedback type $d$ that user $n$ generates while visiting $\mathbf{C}_{p}$. Our goal is to rank candidate pairs of group and MVD configurations. Recall Figure 1(a) illustrating an MVD configuration $\mathrm{C}_{p}$ for a group $g_{p}$ with $V_{p}=\{x, y, z\}$ and $E_{p}=\{(x, y),(y, z)\}$. Sequence $\mathrm{C}_{p}(y,:)=\left\langle i_{1}, i_{3}, i_{4}\right\rangle$ shows the primary view of $y$ on items $i_{1}, i_{3}$, and $i_{4}$ in order. As shown, $x$ and $y$ may discuss $i_{1}$ at slot 1 , while the primary view of $z$ is $i_{2}$. Note that $y$ can switch to the group view with $z$ to discuss $i_{2}$.

\subsection{Configuration Feature Extraction}

In the following, we first introduce the item-level and configurationlevel features, respectively, in order to better characterize MVD configurations according to Marketing theories for alleviating coldstart issue in 3.2. We also extract social behavior features for quantifying extraversion of each user in Section 3.3.

Item-level features (ILF). Users may be attracted by certain appearances of items [10]. Therefore, we extract the $3 D$ shape embedding of items [32] as a feature. Moreover, the purchase rate of highly personal products (e.g., accessories) is usually lower in group shopping [37]. In contrast, new arrivals and advertised items usually trigger Marketing Buzz and social interactions, as well as the number of purchases [12]. Therefore, we extract 1) the product category and 2) the search frequency on Web for each item. For textual features, we extract the word embedding [19] from the product description provided by vendors.

Configuration-level features (CLF). Research reveals that placing correlated products nearby usually increases the purchase rate [7]. Therefore, we extract the average similarity of nearby products in the primary view. In addition, we extract four statistical measurements of configurations: 1) the number of MVD items, 2) the maximal and minimal number of distinct items displayed in a single slot, 3) the average number of distinct items in a single slot for each configuration, and 4) the total number of categories, which represents the diversity of each configuration.

Social behavior features (SBF). Extroverts are more gregarious, outgoing, and talkative in nature [4]. Therefore, we extract 1) the number of times a user switches to other views, 2) the acoustic sentiment, 3) the interaction percentage within groups, 4) the conversation duration in one MVD recommendation, and 5) the number of times a user talks to others for each user.

\subsection{Social-Aware Multi-Feedback Coupled Tensor Factorization}

Thanks to the digital environment, it is convenient to record heterogeneous feedback and extract various features from 3D logged data, e.g., view and touch. Equipped with the heterogeneous feedback, as well as ILF and CLF, the goal is to extract the latent features for each user and MVD configuration to learn the ranking score. As the heterogeneous feedback from users for the configurations is naturally represented as a tensor, we design a new tensor factorization model for learning the MVD personal satisfaction of MVD configurations and companions for a user. Specifically, given $\mathcal{T}, \mathrm{CP}$ factorization [1] of a tensor $\mathcal{T}$ is defined as follows.

$$
\mathcal{T} \approx O \times_{1} \mathrm{U} \times_{2} \mathrm{~V} \times{ }_{3} \mathrm{~W},
$$

where $O \in \mathbb{R}^{\alpha^{3}}$ is a diagonal tensor (i.e., $O(n, m, d)=1$ if $n=$ $m=d$, and 0 otherwise), and $\alpha$ represents the dimensionality of latent features. $\mathrm{U} \in \mathbb{R}^{N \times \alpha}, \mathbf{V} \in \mathbb{R}^{M \times \alpha}$, and $\mathbf{W} \in \mathbb{R}^{D \times \alpha}$ are the latent matrices of users, MVD configurations, and feedback, respectively. $\times_{t}$ denotes the $t$-mode product. For simplicity, let $\mathbf{u}=\mathbf{U}^{\top}(u,:)$ and $\mathbf{p}=\mathbf{V}^{\top}(p,:)$ be the user embedding of user $u$ and MVD configuration embedding of $\mathrm{C}_{p}$, respectively. The MVD personal satisfaction of user $u$ for configuration $\mathrm{C}_{p}$ is represented as follows.

$$
r_{u, p}=\theta^{\top} \mathbf{W}(\mathbf{u} \circ \mathbf{p}),
$$

where $\theta \in \mathbb{R}^{D}$ is employed to learn the contribution of each feedback to the above satisfaction, and $\circ$ is the Hadamard product. In other words, $\mathbf{W}(\mathbf{u} \circ \mathbf{p})$ infers the feedback vector for $u$ visiting $\mathbf{C}_{p}$, and $\theta$ further maps the feedback to $r_{u, p}$.

However, the above approach cannot derive the latent features of new configurations due to the lack of any feedback, i.e., the cold-start problem. Therefore, we further exploit ILF and CLF introduced in Section 3.1 for constructing the feature matrix of $M$ configurations (denoted as $\mathbf{F} \in \mathbb{R}^{\beta \times M}$ ). Let $\mathbf{h}_{p}$ and $\mathbf{i}_{\mathrm{C}_{p}(u, k)}$ denote the CLF vector of $\mathrm{C}_{p}$ and the ILF vector of the item that user $u$ views at slot $k$ in configuration $\mathbf{C}_{p}$, respectively. In addition to $\mathbf{h}_{p}$, we form the configuration features $\mathrm{F}(:, p)$ from ILF, which consists of two parts: i) the primary view vector, which is the concatenation of $k$ item features for each user $u \in V_{p}$; ii) the group view vector, which includes the friends' primary views, as follows.

$$
\mathbf{F}(:, p)=\mathbf{h}_{p} \oplus \sum_{u \in V_{p}}\left(\left[\begin{array}{c}
{ }^{\mathbf{i}_{C_{p}}(u, 1)} \\
\mathbf{i}_{\mathrm{C}_{p}(u, 2)} \\
\vdots \\
\mathbf{i}_{\mathrm{C}_{p}(u, k)}
\end{array}\right]+\sum_{(u, v) \in E_{p}}\left[\begin{array}{c}
\mathbf{i}_{\mathrm{C}_{p}(v, 1)} \\
\mathbf{i}_{\mathrm{C}_{p}(v, 2)} \\
\vdots \\
\mathbf{i}_{\mathrm{C}_{p}(v, k)}
\end{array}\right]\right),
$$

where $\oplus$ is the concatenation operator. In summary, $\mathrm{F}(:, p)$ concatenates CLF and the summation of ILF displayed at each slot based on different combinations of primary views and group views.

However, Eq. (2) does not capture the different importance of group view vectors for different friends. For example, a user $u$ may tend to view identical items with a close friend $v$ for social interactions, so the group view vector of $v$ is more relevant to $u$. In contrast, when the social tightness of $u$ and the others is weak, the user may pay more attention on her primary view. Let $\tau_{u, u, p}$ and $\tau_{u, v, p}$ denote the importance of primary views and group views in configuration $C_{p}$ for user $u$,

$$
\begin{aligned}
\tau_{u, v, p} & =\frac{P r_{u, v}}{P r_{u, u}+\sum_{(u, v) \in E_{p}} P r_{u, v}} \\
\tau_{u, u, p} & =1-\sum_{(u, v) \in E_{p}} \tau_{u, v, p},
\end{aligned}
$$

where $\operatorname{Pr}_{u, u}$ and $\operatorname{Pr}_{u, v}$ represent the likelihood of $u$ to stay at her primary view and the likelihood of $u$ to switch to group view of $v$, respectively. ${ }^{4}$ Therefore, $\tau_{u, u, p}$ and $\tau_{u, u, p}$ are the relative conditional probabilities for $u$ while visiting $\mathrm{C}_{p}$ with $g_{p}$. Accordingly,

\footnotetext{
${ }^{4}$ We can pre-train $\operatorname{Pr}_{u, u}$ and $P r_{u, v}$ with social tightness [3] obtained from online social networks, e.g., Facebook or Twitter.
} 
we can rewrite the feature vector $\mathrm{F}(:, p)$ in Eq. (2) as follows.

$$
\mathbf{F}(:, p)=\mathbf{h}_{p} \oplus \sum_{u \in V_{p}}\left(\tau_{u, u, p}\left[\begin{array}{c}
\mathbf{i}_{\mathrm{C}_{p}(u, 1)} \\
\mathbf{i}_{\mathrm{C}_{p}(u, 2)} \\
\vdots \\
\mathbf{i}_{\mathrm{C}_{p}(u, k)}
\end{array}\right]+\sum_{(u, v) \in E_{p}} \tau_{u, v, p}\left[\begin{array}{c}
\mathbf{i}_{\mathrm{C}_{p}(v, 1)} \\
\mathbf{i}_{\mathrm{C}_{p}(v, 2)} \\
\vdots \\
\mathbf{i}_{\mathrm{C}_{p}(v, k)}
\end{array}\right]\right) .
$$

As we consider both the primary and the group views with different importances in Eq. (4), the interplay of personal interests and social relations are modeled in the feature vector for learning in the following process. Specifically, it is desired for a user to view preferred items in her primary view, but the group views of friends, especially close friends (with large $\tau_{u, v, p}$ ), also affect the user's MVD personal satisfaction.

To facilitate the coupled tensor factorization of the feedback tensor $\mathcal{T}$ and configuration feature matrix $F$, it is necessary to find the relation between $\mathbf{V}$ (latent matrix of configurations from $\mathcal{T}$ ) and $\mathbf{F}$ (directly constructed from ILF and CLF). We introduce a projection matrix $\mathbf{J} \in \mathbb{R}^{\beta \times \alpha}$ such that $\mathbf{V} \approx \mathbf{F}^{\top} \mathbf{J}$. We learn $\mathbf{J}$ to integrate important configuration features in $\mathrm{F}$ and to reduce the difference between $\mathbf{V}$ and $\mathbf{F}^{\mathbf{T}} \mathrm{J}$. The integration not only makes two configuration embeddings with similar contents close to each other in the latent space but also alleviates the cold-start issue, i.e., the embedding of a new configuration without sufficient feedback records can be derived from projecting its ILF and CLF to the shareable feature space. The loss function of the proposed coupled tensor factorization model Configuration Feature Coupled Tensor Factorization (CECON), denoted as $\mathcal{L}_{C E C O N}$, is derived as follows.

$\mathcal{L}_{C E C O N}(\mathbf{U}, \mathbf{V}, \mathbf{W}, \mathbf{J})=\frac{1}{2}\left\|\mathcal{T}-O \times_{1} \mathbf{U} \times_{2} \mathbf{V} \times_{3} \mathbf{W}\right\|^{2}+\frac{\lambda_{1}}{2}\left\|\mathbf{V}-\mathbf{F}^{\top} \mathbf{J}\right\|^{2}$, where the Forbenius norm of a tensor $\mathcal{T}$ is defined as $\|\mathcal{T}\|=$ $\sqrt{\langle\mathcal{T}, \mathcal{T}\rangle}$, and $\lambda_{1}$ controls the contribution of the similarity loss. With $\mathbf{U}, \mathbf{V}$ and $\mathbf{W}$ derived from coupled tensor factorization, we learn the ranking score according to Eq. (1).

\subsection{Ranking MVD Configurations with CECON}

After deriving the latent features of users and configurations with CECON, the goal of the next phase is to propose a learning model for quantifying the MVD personal satisfaction of the candidate MVD configurations. Nevertheless, the learning model is in lack of the ground truth of $r_{u, p}$ since users may not reveal their MVD personal satisfaction of MVD configurations after shopping. Conventional recommenders usually use purchase as the proxy ground truth for MVD personal satisfaction. However, one single kind of feedback may not be sufficient to estimate the MVD personal satisfaction since other interactions (e.g., touching and discussing) may better reflect the MVD personal satisfaction of shopping. We introduce configuration dominance to effectively compare MVD personal satisfaction between two different configurations. Therefore, we formulate the learning of MVD personal satisfaction as a ranking problem and optimize the ranking score by BPR ranking framework (for comparisons) and CECON. Finally, the social norm of extroverts and introverts toward dynamic group change is considered.

Specifically, using different kinds of feedback provides more useful clues of MVD personal satisfaction than using the number of purchases. For example, one MVD configuration has 4 purchases and 8 views, while another one has 5 purchases and 2 views. Although the user slightly purchases more in the latter (4 vs. 5) but she interacts with items more during the visit of the former (12 vs. 7). It is hard to identify which configuration satisfies the user more when the data has heterogeneous feedback. To deal with the implicit feedback, inspired by BPR [22], we learn to rank the MVD configurations by comparing each pair of distinct MVD configurations. We introduce the configuration dominance $>_{u}$ as follows.

$$
\begin{aligned}
& \quad \mathrm{C}_{p}>_{u} \mathrm{C}_{q} \\
& \text { iff } \mathcal{T}(u, p, d) \geq \mathcal{T}(u, q, d), \forall d, \\
& \text { and } \exists d^{*} \text { s.t. } \mathcal{T}\left(u, p, d^{*}\right)>\mathcal{T}\left(u, q, d^{*}\right) .
\end{aligned}
$$

That is, $u$ prefers $\mathrm{C}_{p}$ to $\mathrm{C}_{q}$ if the feedback from $u$ upon any type for $\mathrm{C}_{p}$ is no less than $\mathrm{C}_{q}$. Accordingly, we construct a training dataset of BPR as $D B=\left\{(u, p, q) \mid u \in V, \mathrm{C}_{p}>_{u} \mathrm{C}_{q}\right\}$, where $\mathrm{C}_{p}$ and $\mathrm{C}_{q}$ are distinct MVD configurations visited by $u$.

With configuration dominance, we formulate a new BPR-based ranking loss $\mathcal{L}_{R A N K}$ as follows.

$$
\mathcal{L}_{R A N K}(\mathbf{U}, \mathbf{V}, \mathbf{W}, \theta)=\sum_{(u, p, q) \in D B}-\ln \sigma\left(\rho_{u, p, q} \cdot r_{u, p, q}\right),
$$

where $r_{u, p, q}=r_{u, p}-r_{u, q}$ is the difference of ranking score for a dominant instance $(u, p, q) \in D B, \sigma(\cdot)$ is the sigmoid function that maps $r_{u, p, q}$ to a value between 0 and 1 , and $\ln (\cdot)$ is the $\log -$ likelihood. Moreover, $\rho_{u, p, q}=\sum_{d=1}^{D}[\mathcal{T}(u, p, d)-\mathcal{T}(u, q, d)]$ quantifies the degree of dominance for a dominant instance $(u, p, q)$, i.e., how much more $u$ likes to interact with $\mathrm{C}_{p}$ than with $\mathrm{C}_{q}$. By introducing $\rho_{u, p, q}$ as the confidence of the dominant instance, $\mathcal{L}_{R A N K}$ focuses on correctly ranking the instance with a larger $\rho_{u, p, q}$. For example, for a user $u$, there exist three configurations $\mathrm{C}_{1}, \mathrm{C}_{2}$, and $\mathrm{C}_{3}$ with $\mathrm{C}_{1}>_{u} \mathrm{C}_{2}$ and $\mathrm{C}_{1}>_{u} \mathrm{C}_{3}$. Moreover, $u$ has 25, 1, 22 feedback records while visiting $\mathrm{C}_{1}, \mathrm{C}_{2}$, and $\mathrm{C}_{3}$, respectively. Therefore, the loss function significantly increases if the pair $\left(\mathbf{C}_{1}, \mathbf{C}_{2}\right)$ with $\rho_{u, 1,2}=24$ is incorrectly ranked, while another pair $\left(\mathbf{C}_{1}, \mathbf{C}_{3}\right)$ is with less confidence since $\rho_{u, 1,2}=3$ for $u$.

Moreover, we propose foint $\underline{C} E C O N$ and Configuration Ranking (JOCOR) to learn the MVD personal satisfaction by minimizing $\mathcal{L}_{C E C O N}$ and the pairwise ranking loss $\mathcal{L}_{R A N K}$ jointly.

$$
\begin{aligned}
\mathcal{L}(\Theta) & =\mathcal{L}_{C E C O N}(\mathbf{U}, \mathbf{V}, \mathbf{W}, \mathbf{J})+\mathcal{L}_{R A N K}(\mathbf{U}, \mathbf{V}, \mathbf{W}, \theta) \\
& =\frac{1}{2}\left\|\mathcal{T}-O \times_{1} \mathbf{U} \times_{2} \mathbf{V} \times{ }_{3} \mathbf{W}\right\|^{2}+\frac{\lambda_{1}}{2}\left\|\mathbf{V}-\mathbf{F}^{\top} \mathbf{J}\right\|^{2} \\
& +\sum_{(u, p, q) \in D B}-\ln \sigma\left(\rho_{u, p, q} \cdot r_{u, p, q}\right)+\frac{\lambda_{2}}{2}\|\Theta\|^{2},
\end{aligned}
$$

where $\Theta=\{\mathbf{U}, \mathbf{V}, \mathbf{W}, \mathbf{J}, \theta\}$ and $\lambda_{2}$ controls the sparsity regularization term. Note that minimizing $\mathcal{L}_{C E C O N}$ learns the embeddings from the interplay of users, MVD configurations, and heterogeneous feedback. On the other hand, minimizing $\mathcal{L}_{R A N K}$ incorporates the information of comparisons with configuration dominance in the embeddings. As a result, JOCOR aims to strike a balance between two distinct aspects via a data-driven approach by minimizing two different losses simultaneously.

To address the third challenge regarding complicated interactions during group shopping, we further incorporate the social norm into the proposed model. Specifically, according to previous 
research [4], introverts are inclined to feel anxious when the group members change frequently, which reduces the satisfaction of the activity, but extroverts are more easy-going with the change. Since MVD partitions the group into subgroups based on primary views and social relations in each slot, it is necessary to avoid dramatic subgroup changes for the introverts. For example, an introvert user $u$ may view common items with three friends in slot 1 , and view common items with another three friends in slot 2. $u$ may be overwhelmed due to this great change between two consecutive slots. To find the extraversion of $u$ (denoted as $\kappa(u)$ ) in VR, we take the average of normalized SBF (detailed in Section 3.1) for each user to quantify $\kappa(u) \in[0,1]$. That is, $\kappa(u)$ is large if $u$ is an extrovert. Furthermore, we measure the group dynamic $\delta(u, p)$ of $\mathrm{C}_{p}$ from the perspective of $u$ as follows.

$$
\delta(u, p)=\sum_{i=0}^{k-1} \sum_{(u, v) \in E_{p}(u, i) \ominus E_{p}(u, i+1)} \sigma\left(\mathbf{u}^{\top} \mathbf{v}\right),
$$

where $E_{p}(u, i)$ is the set of social edges of $u$ sharing common items in their primary views in slot $i, \ominus$ is the symmetric difference (disjunctive union) operator for two sets, and $\sigma$ is the sigmoid function. $\delta(u, p)$ increases more when 1$)$ a friend having similar interests (i.e., $\sigma\left(\mathbf{u}^{\top} \mathbf{v}\right)$ is large), or 2) a large number of friends (i.e., $\left|E_{p}(u, i) \ominus E_{p}(u, i+1)\right|$ is large) changes between two consecutive slots. Accordingly, we design a regularization term Group Dynamic Adoption $R_{G D A}$ to regularize the ranking scores.

$$
R_{G D A}=\sum_{\mathrm{C}_{p} \in C_{H}} \sum_{u \in V_{p}} \Delta(u, p) \cdot r_{u, p},
$$

where $\Delta(u, p)=(1-\kappa(u)) \cdot \delta(u, p)$ and $1-\kappa(u)$ discounts the effect of group dynamic $\delta(u, p)$. Therefore, when a user $u$ is an introvert (large $1-\kappa(u)$ ) and the group dynamic is large (large $\delta(u, p)$ ), the MVD personal satisfaction decreases. Incorporating the penalty of the social norm, the final objective function is:

$$
\begin{aligned}
\mathcal{L}(\Theta) & =\frac{1}{2}\left\|\mathcal{T}-O \times_{1} \mathbf{U} \times_{2} \mathbf{V} \times_{3} \mathbf{W}\right\|^{2}+\frac{\lambda_{1}}{2}\left\|\mathbf{V}-\mathbf{F}^{\top} \mathbf{J}\right\|^{2} \\
& +\sum_{(u, p, q) \in D B}-\ln \sigma\left(\rho_{u, p, q} \cdot r_{u, p, q}\right)+\frac{\lambda_{2}}{2}\|\Theta\|^{2} \\
& +\lambda_{3} \sum_{\mathrm{C}_{p} \in C_{H}} \sum_{u \in V_{p}} \Delta(u, p) \cdot r_{u, p},
\end{aligned}
$$

where $\lambda_{3}$ is the weighting hyperparameter controlling $R_{G D A}$.

To minimize $\mathcal{L}(\Theta)$, we exploit the Stochastic Gradient Descent (SGD) algorithm to optimize the model parameters in Section 3.4. Note that the goal of this paper is to correctly quantify the MVD personal satisfaction of users to the configurations and companions. For the recommendation, when $u$ wants to activate a VR shopping activity with friends, given a set of candidates consisting of (group, configuration) pairs, we first derive each user's MVD personal satisfaction with MEIRS. The widely-used Aggregate Voting (AV) mechanism can be adopted to recommend the best (group, configuration) pair. That is, the group and the corresponding configuration with the greatest total satisfaction among the users are recommended.

\subsection{SGD Optimization}

We derive the likelihood function $\ln \left(\sigma\left(\rho_{u, p, q} \cdot r_{u, p, q}\right)\right)$ as follows:

$$
\begin{aligned}
& \frac{\partial \ln \left(\sigma\left(\rho_{u, p, q} \cdot r_{u, p, q}\right)\right)}{\partial \Theta}=\frac{\partial \ln \left(\sigma\left(\varphi_{u, p, q}\right)\right)}{\partial \sigma\left(\varphi_{u, p, q}\right)} \frac{\partial \sigma\left(\varphi_{u, p, q}\right)}{\partial \varphi_{u, p, q}} \frac{\partial \varphi_{u, p, q}}{\partial \Theta} \\
& =\frac{1}{\sigma\left(\varphi_{u, p, q}\right)} \sigma\left(\varphi_{u, p, q}\right)\left(1-\sigma\left(\varphi_{u, p, q}\right)\right) \frac{\partial \varphi_{u, p, q}}{\partial \Theta}=\sigma\left(-\varphi_{u, p, q}\right) \frac{\partial \varphi_{u, p, q}}{\partial \Theta} \\
& =\rho_{u, p, q} \cdot \sigma\left(-\rho_{u, p, q} \cdot r_{u, p, q}\right) \frac{\partial r_{u, p, q}}{\partial \Theta}=\phi_{u, p, q} \frac{\partial r_{u, p, q}}{\partial \Theta},
\end{aligned}
$$

where $\varphi_{u, p, q}$ and $\phi_{u, p, q}$ denote $\rho_{u, p, q} \cdot r_{u, p, q}$ and $\rho_{u, p, q}$. $\sigma\left(-\rho_{u, p, q} \cdot r_{u, p, q}\right)$, respectively. The gradients of $\mathcal{L}$ with respect to $\Theta$ in each iteration are derived as follows.

$$
\begin{aligned}
& \nabla_{\mathbf{u}} \mathcal{L}=\sum_{m=1}^{M} \sum_{d=1}^{D}\left[O \times_{2} \mathbf{V}(m,:) \times_{3} \mathbf{W}(d,:)\right]^{\top} \\
& \text { - }\left[O \times_{1} \mathrm{U}(u,:) \times_{2} \mathrm{~V}(m,:) \times_{3} \mathbf{W}(d,:)-\mathcal{T}(u, m, d)\right] \\
& -\phi_{u, p, q} \cdot\left[(\mathbf{p}-\mathbf{q}) \odot \mathbf{W}^{\top}\right] \theta+\lambda_{2} \mathbf{u} \\
& \left.+\lambda_{3}[\Delta(u, p) \mathbf{p}-\Delta(u, q) \mathbf{q}] \odot \mathbf{W}^{\top}\right] \theta \text {, } \\
& \nabla_{\mathrm{p}} \mathcal{L}=\sum_{n=1}^{N} \sum_{d=1}^{D}\left[O \times_{1} \mathbf{U}(n,:) \times_{3} \mathbf{W}(d,:)\right]^{\top} \\
& \text { - }\left[O \times_{1} \mathrm{U}(n,:) \times_{2} \mathrm{~V}(p,:) \times_{3} \mathbf{W}(d,:)-\mathcal{T}(n, p, d)\right] \\
& -\left[\phi_{u, p, q}+\lambda_{3} \Delta(u, p)\right]\left(\mathbf{W}^{\top} \theta\right) \circ \mathbf{u} \\
& +\lambda_{1}\left(\mathbf{p}-\mathbf{J}^{\top} \mathbf{F}(:, p)\right)+\lambda_{2} \mathbf{p} \text {, } \\
& \nabla_{\mathbf{q}} \mathcal{L}=\sum_{n=1}^{N} \sum_{d=1}^{D}\left[O \times_{1} \mathbf{U}(n,:) \times_{3} \mathbf{W}(d,:)\right]^{\top} \\
& \text { - }\left[O \times_{1} \mathrm{U}(n,:) \times_{2} \mathrm{~V}(q,:) \times_{3} \mathbf{W}(d,:)-\mathcal{T}(n, q, d)\right] \\
& +\left[\phi_{u, p, q}-\lambda_{3} \Delta(u, q)\right]\left(\mathbf{W}^{\top} \theta\right) \circ \mathbf{u} \\
& +\lambda_{1}\left(\mathbf{q}-\mathbf{J}^{\top} \mathbf{F}(:, q)\right)+\lambda_{2} \mathbf{q} \text {, } \\
& \nabla_{\mathrm{J}} \mathcal{L}=-\lambda_{1} \cdot \mathbf{F}\left(\mathbf{V}-\mathbf{F}^{\top} \mathbf{J}\right)+\lambda_{2} \mathbf{J} \text {, } \\
& \nabla_{\mathrm{W}(d,:)} \mathcal{L}=\sum_{n=1}^{N} \sum_{m=1}^{M}\left[O \times_{1} \mathrm{U}(n,:) \times_{2} \mathrm{~V}(m,:)\right] \\
& \text { - }\left[O \times_{1} \mathbf{U}(n,:) \times_{2} \mathbf{V}(m,:) \times_{3} \mathbf{W}(d,:)-\mathcal{T}(n, m, d)\right] \\
& -\phi_{u, p, q} \cdot \theta(d) \cdot[\mathbf{u} \circ(\mathbf{p}-\mathbf{q})]^{\top}+\lambda_{2} \mathbf{W}(d,:) \\
& +\lambda_{3} \cdot \theta(d) \cdot[\mathbf{u} \circ(\Delta(u, p) \mathbf{p}-\Delta(u, q) \mathbf{q})]^{\top} \text {, } \\
& \nabla_{\theta} \mathcal{L}=-\phi_{u, p, q} \cdot \mathbf{W}[\mathbf{u} \circ(\mathbf{p}-\mathbf{q})]+\lambda_{2} \theta \\
& +\lambda_{3} \mathbf{W}[\mathbf{u} \circ(\Delta(u, p) \mathbf{p}-\Delta(u, q) \mathbf{q})],
\end{aligned}
$$

where $\odot$ performs the Hadamard product column-wisely. Although $\Delta(\cdot, \cdot)$ considers the user embeddings $\mathbf{u}$ and $\mathbf{v}$ in Eq. (5), the inner product of $\mathbf{u}$ and $\mathbf{v}$ in each SGD iteration is taken as a constant. Otherwise, the inner product will be minimized and $\mathbf{u}$ and $\mathbf{v}$ are inclined to be separated in the latent feature space, which is definitely not our goal. By iteratively updating the parameters of SGD, tensor factorization loss and pairwise MVD personal satisfaction ranking loss of $u$ and two MVD configurations are minimized jointly.

The time complexity of each iteration in SGD is $O(\alpha D M N)$. However, benefit from CP factorization [1], it is promising to compute the gradients and update each parameter with parallel computing. We show the high efficiency of JOCOR in terms of convergence time 
in Section 4.2. On the other hand, there are $O((N+M+D+\beta) \alpha+D)$ parameters to be learned in JOCOR.

\section{EXPERIMENTAL RESULTS}

In this section, we compare JOCOR with the state-of-the-art personalized recommendation and group recommendation techniques on real datasets. We also conduct a user study to evaluate different ranking methods and collect user feedback for MEIRS.

\subsection{Experiment Setup and Evaluation Plan}

We conduct large-scale experiments on three real datasets for simulation of VR shopping to evaluate the performance of different algorithms. 1) Timik is a VR social gaming dataset with $850 \mathrm{~K}$ users and 12M check-ins [14]. 2) Yelp is a location-based social network (LBSN) with $1.5 \mathrm{M}$ users and $6 \mathrm{M}$ reviews. ${ }^{5}$ ) Foursquare is another LBSN dataset with $2 \mathrm{M}$ users and $280 \mathrm{~K}$ ratings [24]. To simulate MVD configurations for all datasets, similar to [30], we take POIs in the user check-ins within a short period (e.g., two hours) as the items displayed in the primary views. Two users' check-ins at the same POI in the same period represent that they view common items in the same slot. A configuration is constructed by concatenating user check-ins sequentially in multiple time slots. The probability of switching views is approximated by social tightness [9] for all datasets, since users may tend to switch to join a close friend's view more often. In addition to using check-ins as feedback, we extract \{discussion\} on Timik dataset as user feedback, which is the number of messages sent within the period for each slot.

We exploit [32] for extracting 3D shape embeddings as ILF of each POI from Timik. The text descriptions of each POI are also collected by [19] for ILF. We further extract and normalize 1) the total number of messages sent, and 2) the number of friends of each user $u$, to quantify the extraversion $\kappa(u)$. For CLF, we find the correlations, i.e., venues visited in a sequence frequently are regarded as correlated items according to [36] for all datasets. On the other hand, we extract \{tip, review\} for Yelp and \{review for Foursquare as the feedback of items. For both Yelp and Foursquare, the ILF of each POI is extracted from their text descriptions [19] and the average social degree is normalized to be the extraversion. Finally, for each dataset, we extract $6 \mathrm{~K}$ MVD configurations and $2 \mathrm{~K}$ users for training. A group contains 12.3 users on average. It is worth noting that the number of group users in popular VR applications is usually in the small-to-medium scale, e.g., VRChat ${ }^{2}$ allows at most 16 users. We perform five-fold cross-validation, i.e., $80 \%$ data for training and $20 \%$ for validation, to acquire hyper-parameters. Specifically, $\alpha=32, \beta=250, \lambda_{1}=0.1$, and $\lambda_{2}=\lambda_{3}=\eta=0.05$. All algorithms are implemented in an HP DL580 Gen 9 server with four 3.0 GHz Intel CPUs. Each result is reported by averaging over 100 iterations.

We compare JOCOR with the state-of-the-art recommendation technique, including personalized recommender NCF [11], group recommender GBPR [20], and hybrid recommender AGREE [2]. NCF ranks the personal interest by a multi-layer neural network. GBPR employs Matrix Factorization to find group consensus without considering heterogeneous feedback. AGREE learns personal interests and group consensus with a deep attention network. We follow the

\footnotetext{
${ }^{5}$ https://www.yelp.com/dataset/challenge
}

setting in literature to train the above models. GBPR and NCF use check-ins in Timik, ratings in Yelp, and Foursquare as feedback. AGREE transforms the ratings into binary labels (1 if a rating is given and 0 otherwise). In the inference stage, NCF predicts user satisfaction of an item in a configuration. GBPR and AGREE infers user satisfaction of an item with group consensus if the item is shared with friends, or with personal satisfaction otherwise. The MVD personal satisfaction $r_{u, p}$ of a user $u$ and a configuration $\mathrm{C}_{p}$ in the above three approaches is obtained by the average of satisfaction upon the items in her primary view. We also compare with two variants of JOCOR: 1) without the feature matrix F ( JOCOR-N), and ii) without GDA regularization $(\mathcal{F O C O R}-R)$. We evaluate the ranking order of configurations for each user after deriving all the MVD personal satisfaction in the test dataset.

To evaluate configuration recommendation and cold-start issue, we compare the top-K Hit Ratio (HR@K) and Mean Average Precision (MAP@K) of different approaches, where HR@K measures the ratio of the returned top-K ranked configurations belonging to the top-K results in the ground truth, and MAP@K= $\frac{1}{K} \sum_{i=1}^{K} \mathrm{HR} @ \mathrm{i}$ considers the position of a hit by assigning larger weights to the higher-ranked configurations. Furthermore, we compare the MVD personal satisfaction for extroverts and introverts under different settings of groups. Finally, we show the efficiency in terms of training time under different sizes of training datasets. We also conduct a user study by building a VR store prototype with Unity for users wearing hTC VIVE Head Mounted Displays. We recruit 50 participants to visit our VR store, and their social networks are crawled from Facebook. Each group visits the VR store 16 times with different MVD configurations. We record 3D VR data and extract \{view, take, rotation degree, add to cart, purchase\} as heterogeneous feedback [27]. 3D shape embedding is extracted by [32], and the remaining features are manually extracted.

In the following, to show the efficacy of different approaches, we compare JOCOR with the above methods in terms of HR and MAP on large-scale datasets and conduct a user study. The participants are asked to visit the recommended configurations to provide their MVD personal satisfaction and feedback. We analyze the correlation between the ground truth provided by participants and 1) results derived from differnt models, and 2) configuration dominance. Moreover, we investigate the following questions in a questionnaire. Q1) Is the participant satisfied with JOCOR? Q2) Is view switching convenient to join discussions with friends? User feedback is collected in the Likert scale [28], i.e., ranking from 1 to 5 (from very unsatisfactory to very satisfactory). Finally, to analyze the user and configuration embeddings of one shopping group, we visualized them in a $2 \mathrm{D}$ space by [18].

\subsection{Large-Scale Experiment}

Table 1 presents the top-K results of HR@K and MAP@K in Timik, Yelp, and Foursquare. The results manifest that JOCOR outperforms other approaches by at least $31.4 \%$ and $30.8 \%$ in terms of HR and MAP, respectively. The differences between JOCOR and others are statistically significant ( $\mathrm{p}$-value $\leq 0.0003<0.05$ ). The extracted $I L F$, $C L F$, and the extroversion degree are successfully integrated with CECON and GDA. Therefore, JOCOR achieves great improvement (HR: $35.6 \%$ and MAP: $44.0 \%$ ) in Timik. In Yelp, JOCOR outperforms 
Table 1: Top-K performance comparisons on large datasets.

\begin{tabular}{|c|c|c|c|c|c|c|}
\hline \multicolumn{7}{|c|}{ Timik } \\
\hline & \multicolumn{2}{|c|}{$\mathrm{K}=2$} & \multicolumn{2}{|c|}{$\mathrm{K}=3$} & \multicolumn{2}{|c|}{$\mathrm{K}=4$} \\
\hline & HR & MAP & HR & MAP & HR & MAP \\
\hline $\mathrm{NCF}$ & 0.403 & 0.281 & 0.544 & 0.443 & 0.613 & 0.501 \\
\hline AGREE & 0.443 & 0.350 & 0.602 & 0.477 & 0.679 & 0.543 \\
\hline GBPR & 0.283 & 0.245 & 0.312 & 0.288 & 0.493 & 0.321 \\
\hline JOCOR-N & $0.553^{*}$ & $0.433^{*}$ & $0.671^{*}$ & $0.538^{*}$ & $0.751^{*}$ & $0.644^{*}$ \\
\hline JOCOR-R & $0.662^{*}$ & $0.484^{*}$ & $0.700^{*}$ & $0.587^{*}$ & $0.849^{*}$ & $0.701^{*}$ \\
\hline JOCOR & $0.689^{*}$ & $0.516^{*}$ & $0.793^{*}$ & $0.633^{*}$ & $0.921^{*}$ & $0.782^{*}$ \\
\hline \multicolumn{7}{|c|}{ Yelp } \\
\hline & \multicolumn{2}{|c|}{$\mathrm{K}=2$} & \multicolumn{2}{|c|}{$\mathrm{K}=3$} & \multicolumn{2}{|c|}{$\mathrm{K}=4$} \\
\hline & HR & MAP & HR & MAP & HR & MAP \\
\hline $\mathrm{NCF}$ & 0.323 & 0.284 & 0.423 & 0.325 & 0.591 & 0.386 \\
\hline AGREE & 0.395 & 0.294 & 0.517 & 0.422 & 0.628 & 0.464 \\
\hline GBPR & 0.287 & 0.241 & 0.390 & 0.312 & 0.543 & 0.372 \\
\hline JOCOR-N & $\mathbf{0 . 4 7 9}^{*}$ & $0.322^{*}$ & $0.598^{*}$ & $0.455^{*}$ & $0.691^{*}$ & $0.537^{*}$ \\
\hline JOCOR-R & $0.534^{*}$ & $0.351^{*}$ & $0.608^{*}$ & $0.493^{*}$ & $0.757^{*}$ & $0.604^{*}$ \\
\hline JOCOR & $0.610^{*}$ & $0.435^{*}$ & $0.731^{*}$ & $0.552^{*}$ & $0.844^{*}$ & $0.651^{*}$ \\
\hline \multicolumn{7}{|c|}{ Foursquare } \\
\hline & \multicolumn{2}{|c|}{$\mathrm{K}=2$} & \multicolumn{2}{|c|}{$\mathrm{K}=3$} & \multicolumn{2}{|c|}{$\mathrm{K}=4$} \\
\hline & HR & MAP & HR & MAP & HR & MAP \\
\hline $\mathrm{NCF}$ & 0.247 & 0.155 & 0.361 & 0.243 & 0.469 & 0.300 \\
\hline AGREE & 0.264 & 0.169 & 0.379 & 0.258 & 0.486 & 0.311 \\
\hline GBPR & 0.232 & 0.133 & 0.313 & 0.216 & 0.367 & 0.288 \\
\hline JOCOR-N & $0.409^{*}$ & $0.328^{*}$ & $0.430^{*}$ & $0.391^{*}$ & $0.587^{*}$ & $0.400^{*}$ \\
\hline JOCOR-R & $0.411^{*}$ & $0.330^{*}$ & $0.423^{*}$ & $0.382^{*}$ & $0.598^{*}$ & $0.419^{*}$ \\
\hline JOCOR & $0.421^{*}$ & $0.373^{*}$ & $0.498^{*}$ & $0.443^{*}$ & $0.621^{*}$ & $0.467^{*}$ \\
\hline
\end{tabular}

others since it carefully learns from heterogeneous feedback by exploiting configuration dominance. In general, all methods perform worst in Foursquare, but the performance drop of JOCOR is less (27.2\%) difference from Timik than others (at least $44.8 \%$ ) in terms of MAP@2. It is because CECON fully exploits the correlations of successive check-ins extracted for CLF. AGREE outperforms NCF and GBPR more in Timik (10.7\%) than in Yelp (6.2\%) and Foursquare (3.6\%) in terms of HR@4 because users may easily visit virtual POIs with or without friends, indicating that both personal and group check-ins are crucial in VR. In Yelp, the increasing trend of NCF from MAP@2 to MAP@4 (slope: 0.05) is less significant than that from HR@2 to HR@4 (slope: 0.13) in Yelp. It is because personalized recommenders do not consider social interactions but friends in Yelp usually check-in at restaurants together. Therefore, it is hard for NCF to retrieve better configurations early. GBPR has the worst performance because it assumes that users are more satisfied with group consensus than personal interests, which does not always hold. Note that the performance gap between JOCOR and JOCOR-R is larger in Yelp (HR@2: 14.2\% and MAP@2: 23.9\%) than in Foursquare (HR@2: 2.4\% and MAP@2: 2.9\%). The reason is that users often attend a sequence of social activities with friends in Yelp, but users usually visit POIs alone in Foursquare. Therefore, GDA, which captures the group dynamic, is more significant in Yelp. Figure 2 manifests that JOCOR requires less training time to converge than AGREE and NCF because they are built with complex neural networks. JOCOR converges slightly slower than GBPR since it examines heterogeneous feedback and features via CECON but GBPR does not. This difference is greater in Yelp since more feedback is provided.

To examine the ability of JOCOR to address the cold-start issue, the testing configurations are constructed by POIs not in the training datasets. Although we do not have the embeddings of new configurations via CECON, we can utilize the ILF of these POIs
Table 2: Top-K performance comparisons of newconfiguration cold-start on Timik and Yelp.

\begin{tabular}{|c|c|c|c|c|c|c|}
\hline \multicolumn{7}{|c|}{ Timik } \\
\hline & \multicolumn{2}{|c|}{$\mathrm{K}=2$} & \multicolumn{2}{|c|}{$\mathrm{K}=3$} & \multicolumn{2}{|c|}{$\mathrm{K}=4$} \\
\hline & HR & MAP & HR & MAP & HR & MAP \\
\hline $\mathrm{NCF}$ & 0.230 & 0.198 & 0.288 & 0.213 & 0.345 & 0.259 \\
\hline AGREE & 0.233 & 0.199 & 0.291 & 0.227 & 0.388 & 0.295 \\
\hline GBPR & 0.227 & 0.192 & 0.290 & 0.220 & 0.376 & 0.282 \\
\hline JOCOR & $0.406^{*}$ & $0.369^{*}$ & $0.523^{\star}$ & $0.455^{*}$ & $0.637^{*}$ & $0.563^{*}$ \\
\hline \multicolumn{7}{|c|}{ Yelp } \\
\hline & \multicolumn{2}{|c|}{$\mathrm{K}=2$} & \multicolumn{2}{|c|}{$\mathrm{K}=3$} & \multicolumn{2}{|c|}{$\mathrm{K}=4$} \\
\hline & HR & MAP & HR & MAP & HR & MAP \\
\hline $\mathrm{NCF}$ & 0.222 & 0.184 & 0.289 & 0.211 & 0.327 & 0.274 \\
\hline AGREE & 0.209 & 0.164 & 0.267 & 0.193 & 0.318 & 0.222 \\
\hline GBPR & 0.221 & 0.177 & 0.270 & 0.202 & 0.323 & 0.242 \\
\hline JOCOR & $0.389^{*}$ & $0.355^{\star}$ & $0.461^{\star}$ & $0.412^{*}$ & $0.559^{*}$ & $0.501^{*}$ \\
\hline
\end{tabular}

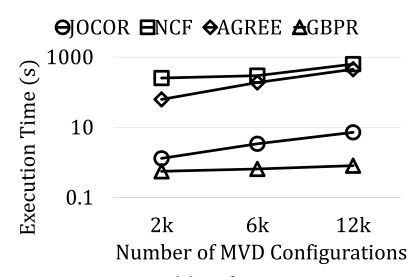

(a) Yelp.

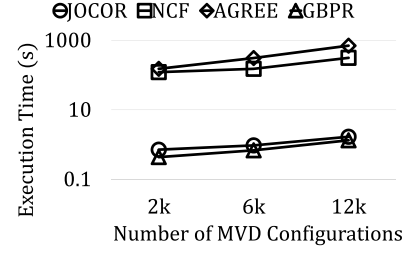

(b) Foursquare.
Figure 2: Execution time on different datasets.

Table 3: Top-K comparisons on user study.

\begin{tabular}{|c|c|c|c|c|c|c|}
\hline \multicolumn{7}{|c|}{ User Study } \\
\hline & \multicolumn{2}{|c|}{$\mathrm{K}=2$} & \multicolumn{2}{|c|}{$\mathrm{K}=3$} & \multicolumn{2}{|c|}{$\mathrm{K}=4$} \\
\hline & HR & MAP & HR & MAP & HR & MAP \\
\hline $\mathrm{NCF}$ & 0.330 & 0.238 & 0.372 & 0.283 & 0.414 & 0.316 \\
\hline AGREE & 0.373 & 0.298 & 0.451 & 0.337 & 0.603 & 0.449 \\
\hline GBPR & 0.329 & 0.276 & 0.433 & 0.342 & 0.512 & 0.402 \\
\hline$\overline{\text { JOCOR-N }}$ & $0.612^{*}$ & $0.533^{*}$ & $0.873^{*}$ & $0.653^{*}$ & $0.951^{*}$ & $0.772^{*}$ \\
\hline JOCOR-R & $0.797^{*}$ & $0.714^{*}$ & $0.889^{*}$ & $0.783^{\star}$ & $0.976^{*}$ & $0.798^{*}$ \\
\hline JOCOR & $0.821^{*}$ & $0.799^{*}$ & $0.973^{*}$ & $0.825^{*}$ & $0.988^{*}$ & $0.843^{*}$ \\
\hline
\end{tabular}

and CLF to construct the content-based feature vectors for new configurations, as shown in Eq. (4). We derive the configuration embeddings by projecting these content-based feature vectors with the projection matrix $\mathrm{J}$. Therefore, JOCOR is able to predict MVD personal satisfaction for cold-start configurations. Table 2 presents the top-K results of HR@K and MAP@K for new configurations in Timik and Yelp. JOCOR outperforms other methods by at least $59.5 \%$ and $72.3 \%$ in terms of HR and MAP for all $\mathrm{K}$ in both datasets. The results manifest that JOCOR effectively transforms the contentbased features (e.g., 3D shape embeddings and POI correlations) to configuration embeddings via $\mathrm{J}$ in CECON. Thus, JOCOR is more robust against cold-start than other baselines. Moreover, JOCOR is generally better in Timik than in Yelp because VR provides more content-based features in nature, which are critical in the cold-start scenario.

\subsection{User Study}

Table 3 manifests that JOCOR outperforms other existing methods in terms of HR (63.8\%) and MAP (87.7\%) because AGREE and GBPR 
Table 4: Correlation analysis of different approaches.

\begin{tabular}{|l|ll|}
\hline & Pearson & Spearman \\
\hline \hline NCF & 0.243 & 0.232 \\
AGREE & 0.342 & 0.333 \\
GBPR & 0.313 & 0.310 \\
\hline JOCOR-N & $\mathbf{0 . 7 9 9}^{*}$ & $\mathbf{0 . 7 8 3}^{\text {* }}$ \\
JOCOR-R & $\mathbf{0 . 8 2 6}^{*}$ & $\mathbf{0 . 8 1 0}^{*}$ \\
JOCOR & $\mathbf{0 . 8 3 4}^{\text {* }}$ & $\mathbf{0 . 8 2 1}^{\text {* }}$ \\
\hline \hline Configuration dominance & $\mathbf{0 . 9 0 1}^{\text {* }}$ & $\mathbf{0 . 8 9 6}^{\text {* }}$ \\
\hline
\end{tabular}
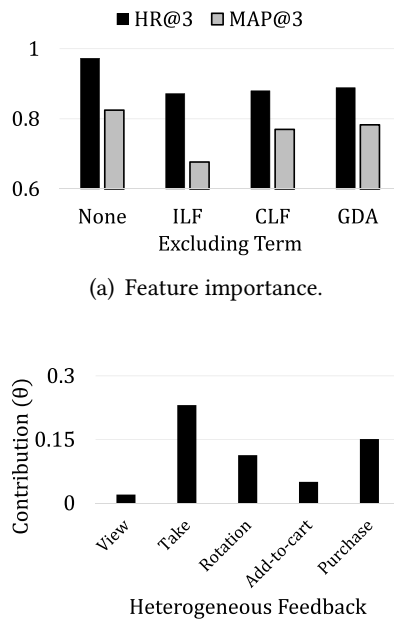

(c) Contribution $\theta$ for each feedback. (a) Feature importance.

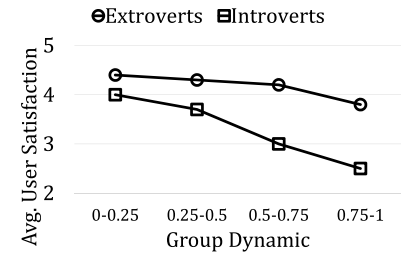

(b) MVD personal satisfaction vs. group dynamic.

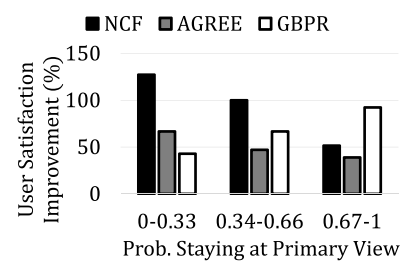

(d) MVD personal satisfaction improvement of JOCOR.

Figure 3: Model analysis.

are not designed for heterogeneous feedback, while NCF deteriorates when many participants are close friends who enjoy discussions. The performance of JOCOR-N dramatically deteriorates comparing with JOCOR (34.1\% in HR@2 and 49.9\% in MAP@2), revealing that ILF and CLF are essential for immersive VR group shopping. An interesting observation is that GBPR performs better than NCF in the user study dataset but worse than NCF in the large-scale experiments, implying the effect of companions in VR shopping is also crucial. We exploit the SVM model to classify introvert and extrovert users with the features discussed in Section 3.3. ${ }^{6}$ The accuracy (88.7\%) indicates these features are discriminative.

To examine the effectiveness of each method, we further analyze the correlation between their results (MVD personal satisfaction derived from the models) and the ground truth (MVD personal satisfaction provided by participants) with Pearson's correlation coefficient and Spearman's rank correlation coefficient. The former is a measure of linear correlation between two variables while the latter is a measure of rank correlation. Table 4 first shows the results of the conventional recommenders. The low correlation coefficients manifest that neither personal nor group recommenders are applicable to recommend MVD configurations. Moreover, NCF has the lowest correlations, which implies that ignoring social interactions

\footnotetext{
${ }^{6}$ The participants are asked to fill the NEO-IP-R questionnaire [5] before the user study. The questionnaire reveals six facets of personality, including Extraversion, which is collected to be the ground truth.
}

in the VR group shopping may result in dissatisfaction. On the other hand, the inferred MVD personal satisfaction of JOCOR (and its variants) is highly correlated to the ground truth and the difference between JOCOR and other baseline methods are statistically significant $(p$-value $\leq 0.004<0.05)$. Furthermore, the results in the last row validate the idea that configuration dominance can serve as an approximation of ground truth for learning.

Figure 3(a) demonstrates the importance of ILF, CLF, and GDA with the leave-one-out strategy, where $K$ is set as 3 . Note that dropping ILF results in the most significant decrement of JOCOR because users tend to interact more with the items that look attractive. On the other hand, dropping $R_{G D A}$ from the objective also lowers the performance. Figure 3(b) further shows the average MVD personal satisfaction of extroverts and introverts under different group dynamic settings. The group dynamic in Eq. (5) for each configuration and user is normalized to a value between 0 and 1 , and then uniformly separated into 4 groups. The slope of MVD personal satisfaction of introverts is steeper than that of extroverts, which implies that introverts are more sensitive to significant group dynamic. Figure 3(c) presents the contribution $\theta$ of each feedback based on the training results. An interesting finding is that "take" is more critical than "purchase" for quantifying MVD personal satisfaction in VR shopping. That is, although users may not purchase items, they enjoy the shopping process and interactions with 3D objects. This also manifests that one single feedback (e.g., purchase) is not sufficient for predicting MVD personal satisfaction. Figure 3(d) presents the percentage of MVD personal satisfaction improvement of JOCOR against different approaches for different group types. Note that when a participant visits the VR mall with a distant group, the value in X-axis, i.e., the probability of staying at her primary view, is greater. JOCOR outperforms GBPR the most (93.3\%) when users are shopping with distant groups, and outperforms NCF the most (127.2\%) when users are with close groups. Note that the improvement against AGREE is slightly higher when the group is closer because the personal interests of displayed items are diverse, which group recommenders fail to address.

The results of the questionnaire reveal that all of the participants are either satisfied $(86.0 \%)$ or very satisfied $(14.0 \%)$ with JOCOR. Only a few participants are unsatisfied (4.0\%) with view switching due to the somewhat complicated interface. However, they all report that friend discussions on products are cornerstones in group shopping. $96.0 \%$ of the participants recognize the importance of MVD in group shopping since it no longer forces everyone to view undesired products while staying with friends. In the interview of the shopping group of 3 females and 1 male (aging from 25 to 28), one female says, "The experience of VR shopping is amazing since MVD allows me to view products I like and intelligently addresses the inconsistent views with easy switching buttons. It is also interesting to find out what my friends like from the group views. From this user study, I feel that our relationships become closer.'

Figure 4(a) illustrates a 2D visualization of user and configuration embeddings of a VR shopping group of 4 users. The observations are two-fold. 1) The embedding of configuration A generating the greatest MVD personal satisfaction (3 fives and 1 four) is close to the 4 users (red dots) in the feature space. This is because the greater MVD personal satisfaction makes the Hadamard product of the user and configuration embeddings also larger, as modeled in 


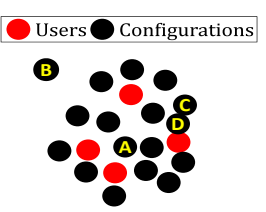

(a) $2 \mathrm{D}$ embeddings of user and configuration.

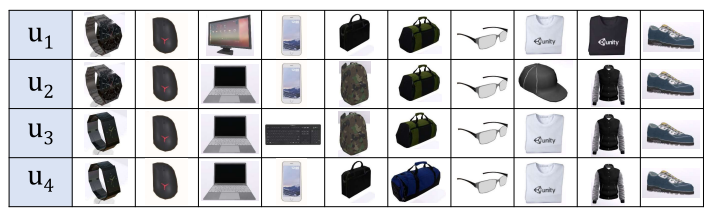

(b) An illustration of configuration C.

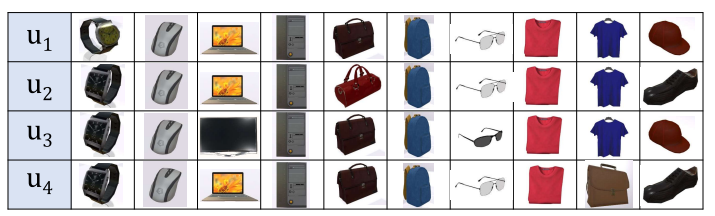

(c) An illustration of configuration D.

Figure 4: An illustrative example for case study.

Eq. (1). Therefore, the two embeddings are inclined to be close in the feature space. In contrast, the least satisfied configuration B (2 ones and 2 twos) is far from the users. 2) Furthermore, Figure 4(b) and 4(c) illustrate configuration $C$ and D in Figure 4(a). Although configurations $\mathrm{C}$ and $\mathrm{D}$ do not share any identical items, the configurations are both comprised of electronics and clothing. As similar configurations are close in the feature space, JOCOR can infer the MVD personal satisfaction of new configurations with existing and similar configurations, and the cold-start issue is thus alleviated.

\section{CONCLUSION}

To the best of our knowledge, there exists no prior research that fully exploits MVD and social interactions for VR group shopping. In this paper, we formulate MECR, propose MEIRS, extract discriminative features, and design a new coupled tensor factorization model CECON combining with a new ranking method JOCOR to rank MVD configurations for each user. Experimental results manifest that JOCOR significantly outperforms personalized and group recommendations by at least $30.8 \%$ in large-scale datasets and $63.3 \%$ in the user study in terms of hit ratio and mean average precision.

\section{ACKNOWLEDGMENT}

This work is supported in part by NSF under grants III-1526499, III-1763325, III-1909323, SaTC-1930941, and CNS-1626432, by MOST in Taiwan through grant 107-2218-E-002-010, 108-2221-E-009-088, 108-2218-E-009-050, and 106-2221-E-009-152-MY3.

\section{REFERENCES}

[1] R. Bro. 1997. Parafac. tutorial and applications. Chemometrics and intelligent laboratory systems (1997).

[2] D. Cao et al. 2018. Attentive group recommendation. In SIGIR

[3] V. Chaoji et al. 2012. Recommendations to boost content spread in social networks. In $W W W$.

[4] H. Cooper et al. 1995. Situation and personality correlates of psychological well-being: Social activity and personal control. Journal of Research in Personality (1995).

[5] P. T Costa and R. McCrae. 1995. Domains and facets: hierarchical personality assessment using the revised neo personality inventory. fournal of personality assessment (1995).

[6] J. Ding et al. 2018. Improving implicit recommender systems with view data. In IJCAI.

[7] W. Gan et al. 2018. Extracting non-redundant correlated purchase behaviors by utility measure. Knowledge-Based Systems (2018).

[8] L. Gao et al. 2018. Recommendation with multi-source heterogeneous information. In $I F C A I$.

[9] A. Goyal et al. 2010. Learning influence probabilities in social networks. In $A C M$ CIKM.

[10] R. He et al. 2016. Sherlock: sparse hierarchical embeddings for visually-aware one-class collaborative filtering. In I7CAI.

[11] X. He et al. 2017. Neural collaborative filtering. In $W W W$.

[12] K. Hewett et al. 2016. Brand buzz in the echoverse. 7. Marketing (2016).
[13] L. Homaeian et al. 2018. Group vs individual: impact of touch and tilt cross-device interactions on mixed-focus collaboration. In $\mathrm{CHI}$.

[14] J. Jankowski et al. 2017. A multilayer network dataset of interaction and influence spreading in a virtual world. Scientific data (2017).

[15] Y. Koren and J. Sill. 2013. Collaborative filtering on ordinal user feedback. In IfCAI.

[16] R. Lissermann et al. 2014. Permulin: mixed-focus collaboration on multi-view tabletops. In $\mathrm{CHI}$.

[17] Z. Lu et al. 2015. Content-based collaborative filtering for news topic recommendation. In $A A A I$.

[18] L. van der Maaten and G. Hinton. 2008. Visualizing data using t-sne. $7 M L R$ (2008).

[19] T. Mikolov et al. 2013. Distributed representations of words and phrases and their compositionality. In NIPS.

[20] W. Pan and L. Chen. 2013. Gbpr: group preference based bayesian personalized ranking for one-class collaborative filtering. In IFCAI.

[21] S. A. P. Parambath et al. 2018. Saga: a submodular greedy algorithm for group recommendation. In $A A A I$.

[22] S. Rendle et al. 2009. Bpr: bayesian personalized ranking from implicit feedback. In UAI.

[23] S. B. Roy et al. 2015. From group recommendations to group formation. In SIGMOD.

[24] M. Sarwat et al. 2013. Umn sarwat foursquare dataset. (2013). http://www-users. cs.umn.edu/ sarwat/foursquaredata/

[25] C.-Y. Shen et al. 2018. On organizing online soirees with live multi-streaming. In $A A A I$.

[26] C.-Y. Shen et al. 2016. Socio-spatial group queries for impromptu activity planning. IEEE TKDE (2016).

[27] V. Sitzmann et al. 2018. Saliency in vr: how do people explore virtual environments? TVCG (2018).

[28] W. Trochim and J. Donnelly. 2001. Research methods knowledge base. (2001).

[29] M. Wan and J. McAuley. 2018. Item recommendation on monotonic behavior chains. In RecSys.

[30] H. Wang et al. 2014. Pgt: measuring mobility relationship using personal, global and temporal factors. In ICDM.

[31] S. Wenzel and M. Benkenstein. 2018. Together always better? the impact of shopping companions and shopping motivation on adolescents' shopping experience. Fournal of Retailing and Consumer Services (2018).

[32] Z. Wu et al. 2015. 3D shapenets: a deep representation for volumetric shapes. In CVPR.

[33] D.-N. Yang et al. 2012. On socio-spatial group query for location-based social networks. In $A C M$ SIGKDD.

[34] H. Yin et al. 2018. Joint event-partner recommendation in event-based social networks. In IEEE ICDE.

[35] H. Yin et al. 2019. Social influence-based group representation learning for group recommendation. In IEEE ICDE.

[36] W. Zhang and J. Wang. 2015. Location and time aware social collaborative retrieval for new successive point-of-interest recommendation. In CIKM.

[37] X. Zhang et al. 2014. An examination of social influence on shopper behavior using video tracking data. f. Marketing (2014).

[38] Y. Zhang et al. 2018. Discrete ranking-based matrix factorization with self-paced learning. In ACM KDD.

[39] T. Zhao et al. 2014. Leveraging social connections to improve personalized ranking for collaborative filtering. In CIKM. 